\title{
Exactly fourteen intrinsically knotted graphs have 21 edges
}

\author{
MiNJUNG LEE \\ HYOUNGJUN KIM \\ HWA JEONG LEE \\ SEUNGSANG $\mathrm{OH}$
}

\begin{abstract}
Johnson, Kidwell, and Michael showed that intrinsically knotted graphs have at least 21 edges. Also it is known that $K_{7}$ and the thirteen graphs obtained from $K_{7}$ by $\nabla Y$ moves are intrinsically knotted graphs with 21 edges. We prove that these 14 graphs are the only intrinsically knotted graphs with 21 edges.
\end{abstract}

$57 \mathrm{M} 25,57 \mathrm{M} 27$

\section{Introduction}

Throughout the article we will take an embedded graph to mean a graph embedded in $R^{3}$. We call a graph $G$ intrinsically knotted if every embedding of the graph contains a knotted cycle. Conway and Gordon [2] showed that $K_{7}$, the complete graph with seven vertices, is an intrinsically knotted graph. A graph $H$ is minor of another graph $G$ if it can be obtained from $G$ by contracting or deleting some edges. An intrinsically knotted graph is minor minimal intrinsically knotted provided no proper minor is intrinsically knotted. Robertson and Seymour [9] proved that there are only finite minor minimal intrinsically knotted graphs, but finding the complete set of them is still an open problem. However, it is well known that $K_{7}$ and the thirteen graphs obtained from this graph by $\nabla Y$ moves are minor minimal intrinsically knotted; see Conway and Gordon [2], and Kohara and Suzuki [6].

A $\nabla Y$ move is an exchanging operation that removes all edges of a triangle $a b c$ and inserts a new vertex $v$ and three edges $v a, v b$ and $v c$ as in Figure 1. Its reverse operation is called a $Y \nabla$ move. Since $\nabla Y$ moves preserve intrinsic knottedness (see Motwani, Raghunathan, and Saran [7]), we will only consider triangle-free graphs in the article.

From the work of Johnson, Kidwell, and Michael [5], it follows that any intrinsically knotted graph consists at least 21 edges. Here is the main theorem. 

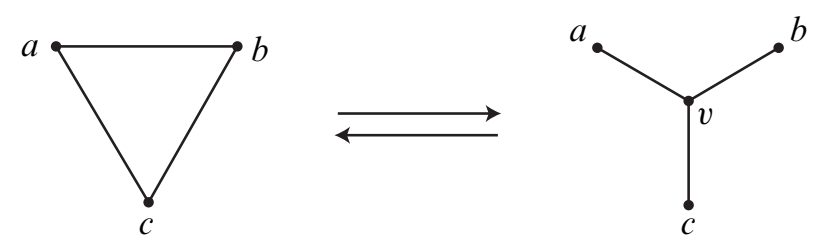

Figure 1: $\nabla Y$ and $Y \nabla$ moves

Theorem 1 The only triangle-free intrinsically knotted graphs with exactly 21 edges are $H_{12}$ and $C_{14}$. ( $H_{12}$ and $C_{14}$ were described by Kohara and Suzuki in [6].)

Kohara and Suzuki [6] found fourteen intrinsically knotted graphs. Goldberg, Mattman, and Naimi [3] constructed twenty graphs derived from $H_{12}$ and $C_{14}$ by $Y \nabla$ moves as in Figure 2, and they showed that these six graphs, $N_{9}, N_{10}, N_{11}, N_{10}^{\prime}, N_{11}^{\prime}$, and $N_{12}^{\prime}$, are not intrinsically knotted. This fact was proved by Hanaki, Nikkuni, Taniyama, and Yamazaki [4] independently. Theorem 1 guarantees that all intrinsically knotted graphs with 21 edges can be obtained from $H_{12}$ and $C_{14}$ by $Y \nabla$ moves. Thus, we have the following theorem.

Theorem 2 The only intrinsically knotted graphs with exactly 21 edges are $K_{7}$ and the thirteen graphs obtained from $K_{7}$ by $\nabla Y$ moves.

This theorem gives us the complete set of fourteen minor minimal intrinsically knotted graphs with 21 edges.

\section{Acknowledgments}

The corresponding author (Seungsang Oh) was supported by the National Research Foundation of Korea (NRF) grant funded by the Korea government (MSIP) number NRF-2014R1A2A1A11050999.

The third author was supported by the National Research Foundation of Korea Grant funded by the Korean Government (NRF-2010-0024630).

\section{Terminology}

From now on let $G=(V, E)$ denote a triangle-free graph with 21 edges. Here $V$ and $E$ denote the sets of all vertices and edges of $G$, respectively. For any two distinct vertices $a$ and $b$, let $\widehat{G}_{a, b}=\left(\widehat{V}_{a, b}, \widehat{E}_{a, b}\right)$ denote the graph obtained from $G$ by deleting two vertices $a$ and $b$, and then contracting an edge incident to a vertex of degree 1 or 


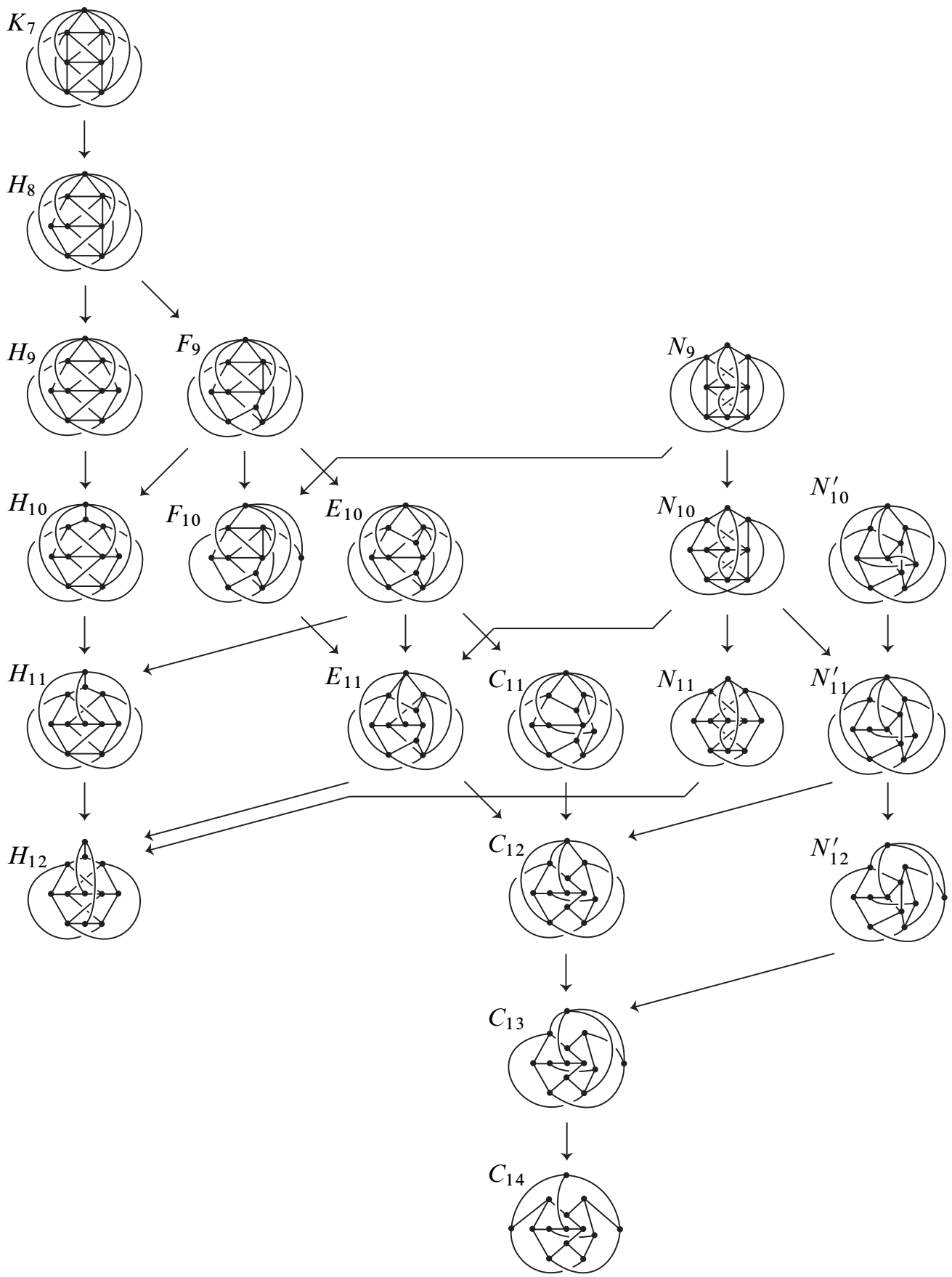

Figure 2: The graph $K_{7}$ and 19 more related graphs, where each arrow represents a $\nabla Y$ move 
2 repeatedly until no vertices of degree 1 or 2 exist. Removing vertices means deleting interiors of all edges incident to these vertices as well as the resulting isolated vertices.

In a graph, the distance between two vertices $a$ and $b$ is the number of edges in the shortest path connecting them and is denoted by $\operatorname{dist}(a, b)$. The degree of a vertex $a$ is denoted by $\operatorname{deg}(a)$. To count the number of edges of $\widehat{G}_{a, b}$, we introduce some notation.

- $E(a)$ is the set of edges which are incident to $a$.

- $V(a)=\{c \in V \mid \operatorname{dist}(a, c)=1\}$.

- $V_{n}(a)=\{c \in V \mid \operatorname{dist}(a, c)=1, \operatorname{deg}(c)=n\}$.

- $V_{n}(a, b)=V_{n}(a) \cap V_{n}(b)$.

- $V_{Y}(a, b)=\left\{c \in V \mid \exists d \in V_{3}(a, b)\right.$ such that $\left.c \in V_{3}(d) \backslash\{a, b\}\right\}$.

First consider the graph $G \backslash\{a, b\}$ for some distinct vertices $a$ and $b$. In this graph each vertex of $V_{3}(a, b)$ has degree 1 , and each vertex of $V_{3}(a), V_{3}(b)$ (not in $V_{3}(a, b)$ ), and $V_{4}(a, b)$ has degree 2 . To derive $\widehat{G}_{a, b}$, we first delete all edges incident to $a$ and $b$ from $G$, and then we also delete the remaining edges incident to $V_{3}(a, b)$, and finally we contract one edge of the remaining pair of edges incident to each vertex of $V_{3}(a)$, $V_{3}(b)$ (not in $V_{3}(a, b)$ ), $V_{4}(a, b)$, and $V_{Y}(a, b)$ as dotted lines in Figure 3(a). Thus, we have the following equation counting the number of edges of $\widehat{G}_{a, b}$ which is called a count equation:

$\left|\widehat{E}_{a, b}\right|=21-|E(a) \cup E(b)|-\left(\left|V_{3}(a)\right|+\left|V_{3}(b)\right|-\left|V_{3}(a, b)\right|+\left|V_{4}(a, b)\right|+\left|V_{Y}(a, b)\right|\right)$.

For short, $N E(a, b)=|E(a) \cup E(b)|$ and $N V_{3}(a, b)=\left|V_{3}(a)\right|+\left|V_{3}(b)\right|-\left|V_{3}(a, b)\right|$. If $a$ and $b$ are adjacent vertices (ie $\operatorname{dist}(a, b)=1$ ), then all of $V_{3}(a, b), V_{4}(a, b)$, and $V_{Y}(a, b)$ are empty because $G$ is triangle-free. Note that this manner of deriving $\widehat{G}_{a, b}$ must be handled in a slightly different way when there is a vertex $c$ in $V$ such that more than one vertex of $V(c)$ are contained in $V_{3}(a, b)$ as in Figure 3(b). In this case, we usually delete or contract more edges incident to $c$, even though $c$ is not in $V_{Y}(a, b)$.

A graph is $n$-apex if one can remove $n$ vertices from the graph to obtain a planar graph. The following lemma gives an important condition for a graph to be not intrinsically knotted.

Lemma 3 [1;8] If $G$ is 2-apex, then $G$ is not intrinsically knotted.

The following two lemmas play an important role for $G$ to be 2 -apex.

Lemma 4 If $\left|\widehat{E}_{a, b}\right| \leq 8$, then $\widehat{G}_{a, b}$ is a planar graph. Thus, $G$ is not intrinsically knotted. 


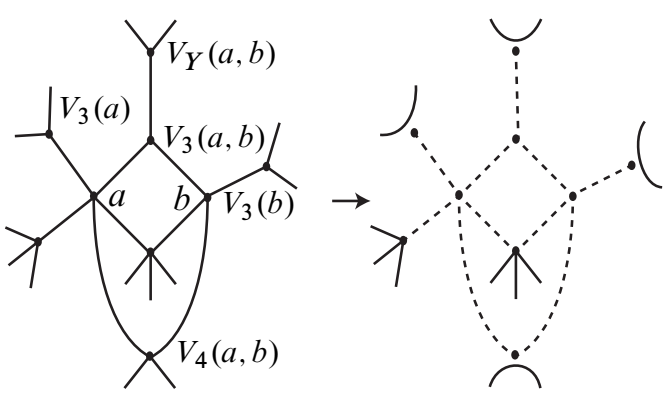

(a)

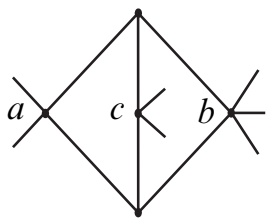

(b)

Figure 3: Deriving $\widehat{G}_{a, b}$

Lemma 5 If $\left|\widehat{E}_{a, b}\right|=9$, then $\widehat{G}_{a, b}$ is either a planar graph or homeomorphic to $K(3,3)$. Furthermore, if $\widehat{G}_{a, b}$ is not homeomorphic to $K(3,3)$, then $G$ is not intrinsically knotted.

The graph $K(3,3)$ is a bipartite graph where each part has three vertices and each vertex is adjacent to every vertex in the opposite part, and so it is a triangle-free graph and every vertex has degree 3 .

To prove Theorem 1, we will show that any triangle-free graph with 21 edges is eventually either a 2-apex or homeomorphic to one of $H_{12}$ or $C_{14}$. Since intrinsically knotted graphs have at least 21 edges [5], it is sufficient to consider simple and connected graphs having no vertex of degree 1 or 2 . Our process is constructing all possible such triangle-free graph $G$ with 21 edges, deleting two suitable vertices $a$ and $b$ of $G$, and then counting the number of edges of $\widehat{G}_{a, b}$. If $\widehat{G}_{a, b}$ has 9 edges or less, we can use Lemma 4 or Lemma 5 in order to show that $G$ is not intrinsically knotted. In the event that $\widehat{G}_{a, b}$ is not planar, we will show that $G$ is homeomorphic to $H_{12}$ or $C_{14}$.

Before describing the proof of Theorem 1, we introduce more notation. Since $G$ is triangle-free, for any vertex $a$ of $G$, no two vertices in $V(a)$ are adjacent. This means that $E(b)$ and $E(c)$ do not contain an edge in common for any two distinct vertices $b$ and $c$ in $V(a)$. We set:

- $E^{2}(a)=\bigcup_{b \in V(a)} E(b)$.

- $E \backslash E^{2}(a)=\left\{e_{1}(a), \ldots, e_{21-n}(a)\right\}$ if $\left|E^{2}(a)\right|=n<21$.

$e_{i}(a)$ is called an extra edge, and the two endpoints of the edge are denoted as $x_{i}(a)$ and $y_{i}(a)$, where $\operatorname{deg}\left(x_{i}(a)\right) \geq \operatorname{deg}\left(y_{i}(a)\right)$. 
In order to visualize $G$, we perform the following steps. First choose a vertex $a$ with the maximal degree among all vertices and draw $E^{2}(a)$. If $\left|E^{2}(a)\right|<21$, draw $E \backslash E^{2}(a)$ apart from $E^{2}(a)$ as in Figure 4(a). Then all vertices of degree 1 of $E^{2}(a)$ and $E \backslash E^{2}(a)$ are merged into some vertices of degree at least 3 without adding new edges as in Figure 4(b). Let $\bar{V}(a)$ denote the set of all such vertices, and let $[\bar{V}(a)]$ denote a sequence of the degrees of vertices in $\bar{V}(a)$ as follows:

- $\bar{V}(a)=V \backslash(V(a) \cup\{a\})=\left\{\bar{v}_{1}(a), \ldots, \bar{v}_{m}(a)\right\}$ with $\operatorname{deg}\left(\bar{v}_{i}(a)\right) \geq \operatorname{deg}\left(\bar{v}_{i+1}(a)\right)$.

- $[\bar{V}(a)]=\left[\operatorname{deg}\left(\bar{v}_{1}(a)\right), \ldots, \operatorname{deg}\left(\bar{v}_{m}(a)\right)\right]$.

- $|[\bar{V}(a)]|=\operatorname{deg}\left(\bar{v}_{1}(a)\right)+\cdots+\operatorname{deg}\left(\bar{v}_{m}(a)\right)$.

The graph in Figure 4(b) is an example satisfying $\operatorname{deg}(a)=5,\left|V_{3}(a)\right|=1,\left|E^{2}(a)\right|=$ 19 , and $[\bar{V}(a)]=[4,4,4,3,3]$.

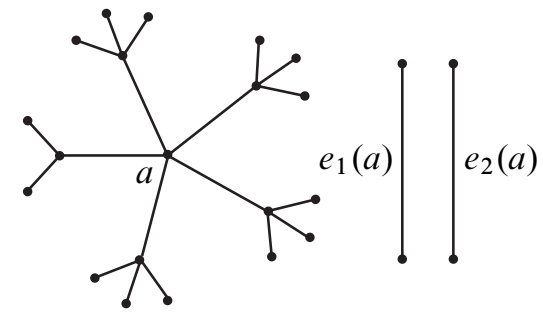

(a)

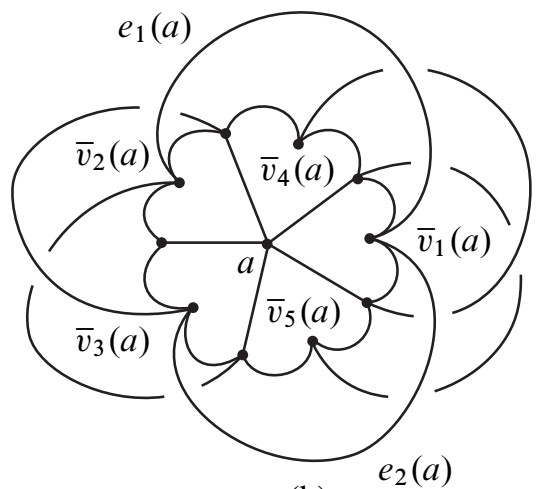

(b)

Figure 4: Visualization of $G$

The remaining three sections of the article are devoted to the proof of Theorem 1. From now on, $a$ denotes one of vertices with maximal degree in $G$. The proof is divided into three parts according to the degree of $a$. In Section 3 we show that any graph $G$ with $\operatorname{deg}(a) \geq 5$ cannot be intrinsically knotted. In Section 4 we show that an intrinsically knotted graph with $\operatorname{deg}(a)=4$ is exactly $H_{12}$. Finally, in Section 5 we show that any intrinsically knotted graph, all of whose vertices have degree 3 , is always $C_{14}$.

\section{$3 \operatorname{deg}(a) \geq 5$}

In this section we will show that for some $a^{\prime}, b^{\prime} \in V$ either $\left|\widehat{E}_{a^{\prime}, b^{\prime}}\right| \leq 8$ or $\left|\widehat{E}_{a^{\prime}, b^{\prime}}\right|=9$, but that $\widehat{G}_{a^{\prime}, b^{\prime}}$ is not homeomorphic to $K(3,3)$ by showing that it contains a vertex of degree more than 3 or a triangle (or sometimes a bigon). Then, as a conclusion, $G$ is not intrinsically knotted by Lemmas 4 and 5. Recall that $G$ has 21 edges, every vertex has degree at least 3 , and $a$ has the maximal degree among them. 


\subsection{Case $\operatorname{deg}(a) \geq 6$ or $\operatorname{deg}(a)=5$ with $\left|V_{3}(a)\right| \geq 4$}

If $\operatorname{deg}(a) \geq 6$, then $\left|V_{3}(a)\right| \geq 3$. Let $c$ be any vertex in $V_{3}(a)$. Choose a vertex $b$ which has the maximal degree among $V(c) \backslash\{a\}$. Then $|E(b)|+\left|V_{Y}(a, b)\right| \geq 4$, since $\left|V_{Y}(a, b)\right| \geq 1$ when $\operatorname{deg}(b)=3$. Note that $\left|V_{3}(b)\right| \geq\left|V_{3}(a, b)\right|$. By the count equation, $\left|\widehat{E}_{a, b}\right| \leq 8$ in $\widehat{G}_{a, b}$.

Suppose that $\operatorname{deg}(a)=5$ and $\left|V_{3}(a)\right| \geq 4$. The proof is similar to the previous paragraph.

\subsection{Case $\operatorname{deg}(a)=5$ and $\left|V_{3}(a)\right|=3$}

Let $b$ and $c$ be two vertices of $V(a) \backslash V_{3}(a)$. First, suppose that both of them have degree 5. Then $N E(a, b)=9$ and $\left|V_{3}(a)\right|=3$, so $\left|\widehat{E}_{a, b}\right| \leq 9$. Furthermore, the vertex $c$ has degree 4 in $\widehat{G}_{a, b}$, so it follows that $\widehat{G}_{a, b}$ is not homeomorphic to $K(3,3)$. Thus, $G$ is not intrinsically knotted by Lemma 5 .

Now assume that one of them, say $b$, has degree 4 . If $V(b) \backslash\{a\}$ consists of three vertices, all of which are of degree 3 , then $N E(a, b)=8$ and $N V_{3}(a, b)=6$, so $\left|\widehat{E}_{a, b}\right| \leq 7$. If not, let $d$ be a vertex of $V(b)$ which has degree at least 4 . Then $N E(a, d) \geq 9,\left|V_{3}(a)\right|=3$, and $\left|V_{4}(a, d)\right| \geq 1$, because $V_{4}(a, d) \ni b$. This implies that $\left|\widehat{E}_{a, d}\right| \leq 8$.

\subsection{Case $\operatorname{deg}(a)=5$ and $\left|V_{3}(a)\right|=0$}

First, suppose that $V(a)$ contains a vertex of degree 5, say $c$. Since $G$ has 21 edges, the other four vertices of $V(a)$ have degree 4 . By the previous cases, it is sufficient to suppose that $\left|V_{3}(c)\right| \leq 2$. So $V(c) \backslash\{a\}$ has at least two vertices, say $b$ and $d$, of degree 4 or 5 . Since $\left|E^{2}(a)\right|=21$ and $G$ is triangle-free, all edges of $E(b)$ must be incident to different vertices of $V(a)$, so $\left|V_{4}(a, b)\right| \geq 3$. This implies that $\left|\widehat{E}_{a, b}\right| \leq 9$. Since $\widehat{G}_{a, b}$ has the vertex $d$ of degree at least 4 , it follows that $\widehat{G}_{a, b}$ is not homeomorphic to $K(3,3)$.

Now, assume that all vertices of $V(a)$ have degree 4 , giving $\left|E^{2}(a)\right|=20$. Let $e_{1}(a)$ be the extra edge and recall that two endpoints of $e_{1}(a)$ are $x_{1}(a)$ and $y_{1}(a)$ with $\operatorname{deg}\left(x_{1}(a)\right) \geq \operatorname{deg}\left(y_{1}(a)\right)$. Since $G$ is triangle-free, all edges of $E\left(x_{1}(a)\right) \cup E\left(y_{1}(a)\right)$ except $e_{1}(a)$ must be incident to different vertices of $V(a)$. Thus the degrees of $x_{1}(a)$ and $y_{1}(a)$ must be either 4 and 3 , or 3 and 3 , respectively. If $\operatorname{deg}\left(x_{1}(a)\right)=4$, then $\left|V_{4}\left(a, x_{1}(a)\right)\right|=3$ and $\left|V_{3}\left(x_{1}(a)\right)\right|=1$, so $\left|\widehat{E}_{a, x_{1}(a)}\right|=8$. If not, $[\bar{V}(a)]$ is either $[5,3,3,3,3]$ or $[4,4,3,3,3]$, because $|[\bar{V}(a)]|=17$. Thus $\bar{v}_{1}(a)$ has degree 5 or 4 and differs from $x_{1}(a)$ and $y_{1}(a)$, so $\left|V_{4}\left(a, \bar{v}_{1}(a)\right)\right| \geq 4$. Therefore, $\left|\widehat{E}_{a, \bar{v}_{1}(a)}\right| \leq 8$. 


\subsection{Case $\operatorname{deg}(a)=5$ and $\left|V_{3}(a)\right|=1$}

In this case, $V(a)$ contains four vertices of degree 4 or 5 . Let $n$ be the number of such vertices of degree 4 , and so we have $4-n$ vertices of degree 5 , where $n=2,3,4$. This implies that $\left|E^{2}(a)\right|=21+(2-n)$, and $n-2$ extra edges exist. If $\bar{V}(a)$ contains a vertex $\bar{v}_{1}(a)$ of degree 5 , then five edges of $E\left(\bar{v}_{1}(a)\right)$ are extra edges or incident to different vertices in $V(a)$. For any of the above $n$, at least two among these edges are incident to vertices of degree 4 in $V(a)$. Then $N E\left(a, \bar{v}_{1}(a)\right)=10,\left|V_{3}(a)\right|=1$, and $\left|V_{4}\left(a, \bar{v}_{1}(a)\right)\right| \geq 2$, implying $\left|\widehat{E}_{a, \bar{v}_{1}(a)}\right| \leq 8$.

Now, suppose that $\bar{V}(a)$ contains vertices of degree 3 or 4 only. If $n=2,|[\bar{V}(a)]|=16$, and so $[\bar{V}(a)]$ is either $[4,4,4,4]$ or $[4,3,3,3,3]$. For any vertex $b$ in $V_{5}(a)$, four edges of $E(b)$ must be incident to different vertices of $\bar{V}(a)$. Indeed, these four edges are incident to four vertices of degree 4 , or at least three edges among them are incident to vertices of degree 3 in $\bar{V}(a)$. This means that the vertex $b$ has degree 5 with either $V_{3}(b)=0$ or $V_{3}(b) \geq 3$. Both cases are dealt with in previous cases 3.3, 3.1, and 3.2.

If $n=3,|[\bar{V}(a)]|=17$, and so $[\bar{V}(a)]=[4,4,3,3,3]$. Let $V_{5}(a)=\{b\}$. To avoid the case 3.2, four edges of $E(b)$ must be incident to two vertices of degree 4 and two vertices of degree 3 in $\bar{V}(a)$, which are $\bar{v}_{1}(a), \bar{v}_{2}(a), \bar{v}_{3}(a)$, and $\bar{v}_{4}(a)$. Then there is a vertex $c$ of $V_{4}(a)$ such that at most one edge of $E(c)$ is incident to $\bar{v}_{3}(a)$ and $\bar{v}_{4}(a)$, ie two edges of $E(c)$ are incident to $\bar{v}_{1}(a), \bar{v}_{2}(a)$, or $\bar{v}_{5}(a)$. This implies that $N E(b, c)=9$ and $N V_{3}(b, c)+\left|V_{4}(b, c)\right| \geq 4$, implying $\left|\widehat{E}_{b, c}\right| \leq 8$.

Finally, if $n=4,|[\bar{V}(a)]|=18$, and so $[\bar{V}(a)]$ is either $[4,4,4,3,3]$ or $[3,3,3,3,3,3]$. Recall that two extra edges exist. In the former case let $\left\{\bar{v}_{1}(a), \bar{v}_{2}(a), \bar{v}_{3}(a)\right\}$ be the three vertices of degree 4 in $\bar{V}(a)$. For each $i=1,2,3$, if more than two edges of $E\left(\bar{v}_{i}(a)\right)$ are incident to $V_{4}(a)$, then $N E\left(a, \bar{v}_{i}(a)\right)=9,\left|V_{3}(a)\right|=1$, and $\left|V_{4}\left(a, \bar{v}_{i}(a)\right)\right| \geq 3$, implying $\left|\widehat{E}_{a, \bar{v}_{i}(a)}\right| \leq 8$. So, each of at least two edges of $E\left(\bar{v}_{i}(a)\right)$ must be either incident to the unique vertex of $V_{3}(a)$ or an extra edge. Since $G$ is triangle-free, one of three vertices, say $\bar{v}_{1}(a)$, has the property that $E\left(\bar{v}_{1}(a)\right)$ contains both extra edges, and $V\left(\bar{v}_{1}(a)\right)$ and $V\left(\bar{v}_{i}(a)\right)$ for each $i=2,3$ cannot share a vertex in $V(a)$. This implies that $V\left(\bar{v}_{2}(a)\right)$ and $V\left(\bar{v}_{3}(a)\right)$ coincide as in Figure 5(a). Then $N E\left(\bar{v}_{2}(a), \bar{v}_{3}(a)\right)=8$, and either $\left|V_{4}\left(\bar{v}_{2}(a), \bar{v}_{3}(a)\right)\right|=4$ or $\left|V_{4}\left(\bar{v}_{2}(a), \bar{v}_{3}(a)\right)\right|=3$ and $\left|V_{3}\left(\bar{v}_{2}(a)\right)\right|=1$. Thus, $\left|\widehat{E}_{\bar{v}_{2}(a), \bar{v}_{3}(a)}\right| \leq 9$. In $\widehat{G}_{\bar{v}_{2}(a), \bar{v}_{3}(a)}$ the vertex $a$ still has degree 4 or 5 so that $\widehat{G}_{\bar{v}_{2}(a), \bar{v}_{3}(a)}$ is not homeomorphic to $K(3,3)$.

In the latter case, let $V_{4}(a)=\left\{b_{1}, b_{2}, b_{3}, b_{4}\right\}$. We claim that for some $i, j=1,2,3,4$, $\left|V_{3}\left(b_{i}, b_{j}\right)\right| \leq 1$. Suppose not; that is, $\left|V_{3}\left(b_{i}, b_{j}\right)\right| \geq 2$ for all combinations of $i$ and $j$. By some combinatorics we can derive that all 12 edges of $E\left(b_{1}\right) \cup E\left(b_{2}\right) \cup$ $E\left(b_{3}\right) \cup E\left(b_{4}\right) \backslash E(a)$ are incident to only four vertices of $\bar{V}(a)$ as in Figure 5(b). 
This means that two extra edges must be incident to the remaining two vertices of $\bar{V}(a)$ at both endpoints. But a bigon is not allowed. Therefore, without loss of generality, $\left|V_{3}\left(b_{1}, b_{2}\right)\right| \leq 1$. Then $N E\left(b_{1}, b_{2}\right)=8$ and $N V_{3}\left(b_{1}, b_{2}\right) \geq 5$, implying $\left|\widehat{E}_{b_{1}, b_{2}}\right| \leq 8$.

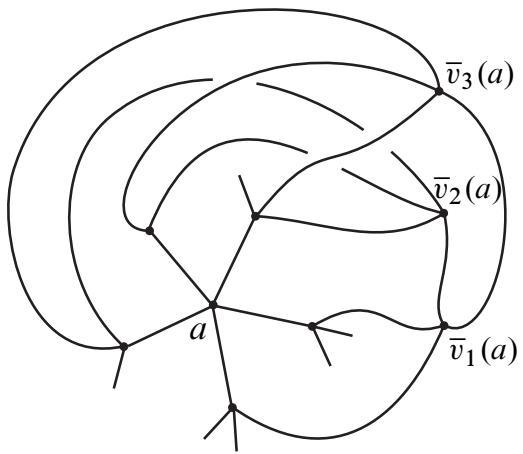

(a)

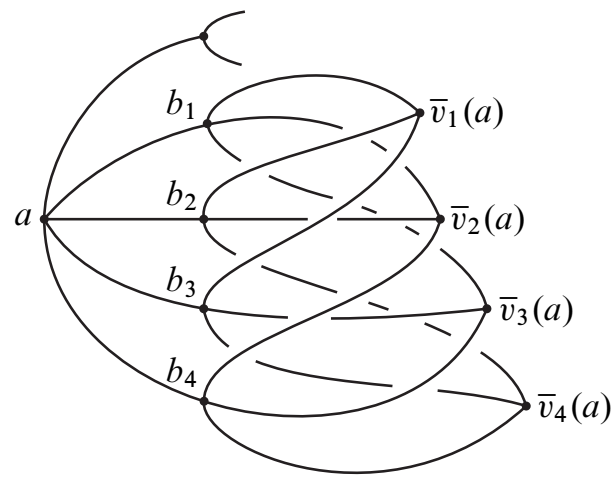

(b)

Figure 5: $[4,4,4,3,3]$ and $[3,3,3,3,3,3]$ cases

\subsection{Case $\operatorname{deg}(a)=5$ and $\left|V_{3}(a)\right|=2$}

If $V(a)$ contains a vertex of degree 5 , say $b$, then the previous four cases guarantee that we only consider that $\left|V_{3}(b)\right|=2$, so $N V_{3}(a, b)=4$, which implies $\left|\hat{E}_{a, b}\right|=8$. Therefore we assume that $V(a)$ contains three vertices of degree 4 . In this case three extra edges exist. Since $|[\bar{V}(a)]|=19,[\bar{V}(a)]$ is one of $[5,5,5,4],[5,5,3,3,3]$, $[5,4,4,3,3],[4,4,4,4,3]$, or $[4,3,3,3,3,3]$.

If, for some vertex $\bar{v}_{i}(a)$ with degree 5 , one edge of $E\left(\bar{v}_{i}(a)\right)$ is incident to $V_{4}(a)$, then $N E\left(a, \bar{v}_{i}(a)\right)=10,\left|V_{3}(a)\right|=2$, and $\left|V_{4}\left(a, \bar{v}_{i}(a)\right)\right| \geq 1$, implying $\left|\widehat{E}_{a, \bar{v}_{i}(a)}\right| \leq 8$. Thus, three edges of $E\left(\bar{v}_{i}(a)\right)$ are extra edges and the remaining two edges are incident to $V_{3}(a)$. In the first two cases, [5, 5, 5,4] and [5, 5, 3, 3, 3], both $E\left(\bar{v}_{1}(a)\right)$ and $E\left(\bar{v}_{2}(a)\right)$ share three extra edges, but $G$ does not have a bigon. In the third case, [5, 4, 4, 3, 3], $E\left(\bar{v}_{1}(a)\right)$ contains three extra edges and one of these extra edges must be incident to $\bar{v}_{4}(a)$ or $\bar{v}_{5}(a)$, both of which have degree 3 . Then $N E\left(a, \bar{v}_{1}(a)\right)=10$ and $N V_{3}\left(a, \bar{v}_{1}(a)\right) \geq 3$, implying $\left|\widehat{E}_{a, \bar{v}_{1}(a)}\right| \leq 8$.

If, for some vertex $\bar{v}_{i}(a)$ with degree 4 , two edges of $E\left(\bar{v}_{i}(a)\right)$ are incident to $V_{4}(a)$, then $N E\left(a, \bar{v}_{i}(a)\right)=9,\left|V_{3}(a)\right|=2$, and $\left|V_{4}\left(a, \bar{v}_{i}(a)\right)\right| \geq 2$, implying $\left|\widehat{E}_{a, \bar{v}_{i}(a)}\right| \leq$ 8. Thus, at most one edge of $E\left(\bar{v}_{i}(a)\right)$ is incident to $V_{4}(a)$. In the fourth case, $[4,4,4,4,3]$, at least twelve among sixteen edges incident to four vertices of degree 4 in $\bar{V}(a)$ are not incident to $V_{4}(a)$. This is impossible because there are only two vertices in $V_{3}(a)$ and three extra edges. In the last case, [4, 3, 3, 3, 3, 3], since only one 
edge of $E\left(\bar{v}_{1}(a)\right)$ is possibly incident to $V_{4}(a)$, there is a vertex $b$ in $V_{4}(a)$ such that three edges of $E(b)$ are incident to vertices of degree 3 in $\bar{V}(a)$. Then $N E(a, b)=8$ and $N V_{3}(a, b) \geq 5$, implying $\left|\widehat{E}_{a, b}\right| \leq 8$.

\section{$4 \operatorname{deg}(a)=4$}

Since $|V|=\left|V_{4}\right|+\left|V_{3}\right|$ and $4\left|V_{4}\right|+3\left|V_{3}\right|=2|E|$, the pair $\left(\left|V_{4}\right|,\left|V_{3}\right|\right)$ has three choices: $(3,10),(6,6)$, and $(9,2)$. Here, $V_{n}$ denotes the set of vertices of degree $n$. As in the preceding section, we will show that for some $a^{\prime}, b^{\prime} \in V$ either $\left|\widehat{E}_{a^{\prime}, b^{\prime}}\right| \leq 8$ or $\left|\widehat{E}_{a^{\prime}, b^{\prime}}\right|=9$, but $\widehat{G}_{a^{\prime}, b^{\prime}}$ is not homeomorphic to $K(3,3)$, implying that $G$ is not intrinsically knotted. But one exception occurs so that $G$ can possibly be $H_{12}$ when $\left(\left|V_{4}\right|,\left|V_{3}\right|\right)=(6,6)$.

\subsection{Case $\left(\left|V_{4}\right|,\left|V_{3}\right|\right)=(3,10)$}

First suppose that $V_{4}$ has a vertex $a$ such that all four vertices of $V(a)$ have degree 3 . Let $b_{1}$ and $b_{2}$ be the other vertices of $V_{4}$. For each $i=1,2, N E\left(a, b_{i}\right)=8$. If there is a vertex of $V_{3}\left(b_{i}\right)$ which is not contained in $V(a)$, then $N V_{3}\left(a, b_{i}\right) \geq 5$, implying $\left|\widehat{E}_{a, b_{i}}\right| \leq 8$. Thus each vertex of $V\left(b_{1}\right)$ is the vertex $b_{2}$ or contained in $V(a)$, and similarly for $b_{2}$. This implies that the number of vertices of $V_{3}$ which have distance 1 or 2 from the vertex $a$ is at most 6 . Take a vertex $c$ of $V_{3}$ with distance at least 3 from $a$. Since each vertex of $V(c)$ is neither $b_{1}$ nor $b_{2}$, it has degree 3 . Thus $N E(a, c)=7$ and $N V_{3}(a, c) \geq 7$, implying $\left|\widehat{E}_{a, c}\right| \leq 7$.

Now, we only need to consider the case that each vertex of $V_{4}$ is adjacent to at least one vertex of degree 4 . Then, without loss of generality, we have vertices $a, b$ and $c$ of $V_{4}$ such that $V(b)$ contains $a$ and $c$. If $V_{3}(a)$ and $V_{3}(c)$ do not coincide, then $\left|V_{4}(a, c)\right|=1$ and $N V_{3}(a, c) \geq 4$, implying $\left|\widehat{E}_{a, c}\right| \leq 8$. If $V_{3}(a)$ and $V_{3}(c)$ coincide and $\left|V_{Y}(a, c)\right| \geq 2$, then $\left|V_{4}(a, c)\right|=1$ and $N V_{3}(a, c)=3$, implying $\left|\widehat{E}_{a, c}\right| \leq 7$. If not, for the unique vertex $d$ of $V_{Y}(a, c), V_{3}(a)=V_{3}(c)=V(d)$. Then, for a vertex $b^{\prime}$ of $V_{3}(b), V_{3}\left(b^{\prime}\right)$ is disjoint from $V_{3}(a)$. Thus $N E\left(a, b^{\prime}\right)=7, N V_{3}\left(a, b^{\prime}\right)=5$, and $\left|V_{4}\left(a, b^{\prime}\right)\right|=1$, implying $\left|\widehat{E}_{a, b^{\prime}}\right| \leq 8$.

\subsection{Case $\left(\left|V_{4}\right|,\left|V_{3}\right|\right)=(6,6)$}

Consider the subgraph $H$ of $G$ consisting of all edges whose both end vertices have degree 4 . Since $G$ has six vertices of degree 3 and the same number of vertices of degree $4, H$ is not empty set.

Claim 1 If $H$ has a vertex of degree 1 , then $G$ is not intrinsically knotted. 
Proof Suppose that $H$ has a vertex $a$ of degree 1 . Let $b$ be the unique vertex of degree 4 in $V(a)$. If $\left|V_{3}(b)\right|=3$, then $N E(a, b)=7$ and $N V_{3}(a, b)=6$, implying $\left|\widehat{E}_{a, b}\right| \leq 8$. Thus, there is another vertex $c$ of $V_{4}(b)$, and so we let $V(c)=\left\{b, d_{1}, d_{2}, d_{3}\right\}$.

First, assume that $\left|V_{3}(c)\right|=0$. So the two vertices of $V(b) \backslash\{a, c\}$ must have degree 3, because the six vertices $a, b, c, d_{1}, d_{2}$, and $d_{3}$ in $V_{4}$ are all different. Thus $N E(a, b)=7$ and $N V_{3}(a, b)=5$, so $\left|\widehat{E}_{a, b}\right| \leq 9$. Since $\widehat{G}_{a, b}$ has another vertex $d_{1}$ of degree 4 , it follows that $\widehat{G}_{a, b}$ is not homeomorphic to $K(3,3)$.

Second, assume that $\left|V_{3}(c)\right|=1$, say $d_{1} \in V_{3}(c)$. If $d_{1}$ is not one of the vertices in $V(a)$, then $N E(a, c)=8$ and $N V_{3}(a, c)+\left|V_{4}(a, c)\right|=5$, implying $\left|\widehat{E}_{a, c}\right| \leq 8$. So we may assume that $d_{1}$ is in $V(a)$ and let $V\left(d_{1}\right)=\left\{a, c, v_{1}\right\}$. If $v_{1}$ has degree 3 , then $N V_{3}(a, c)+\left|V_{4}(a, c)\right|=4$ and $V_{Y}(a, c)=\left\{v_{1}\right\}$, implying $\left|\widehat{E}_{a, c}\right| \leq 8$. Otherwise $v_{1}$ has degree 4 and it is different from $d_{2}$ and $d_{3}$. For any $i=2,3$, each vertex of $V\left(d_{i}\right) \backslash\{c\}$ either has degree 3 or is $v_{1}$. Thus $N E\left(d_{2}, d_{3}\right)=8$ and $N V_{3}\left(d_{2}, d_{3}\right)+\left|V_{4}\left(d_{2}, d_{3}\right)\right| \geq 4$, implying $\left|\widehat{E}_{d_{2}, d_{3}}\right| \leq 9$. But $\widehat{G}_{d_{2}, d_{3}}$ has a triangle containing vertices $a, b$ and $d_{1}$. See Figure 6(a).

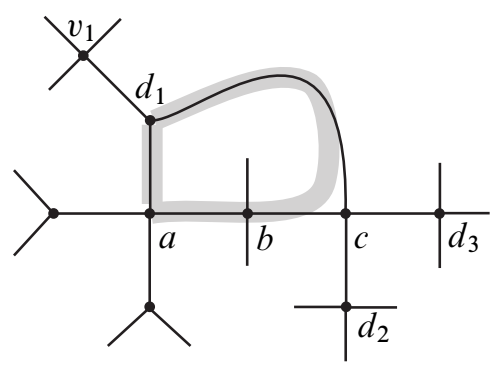

(a)

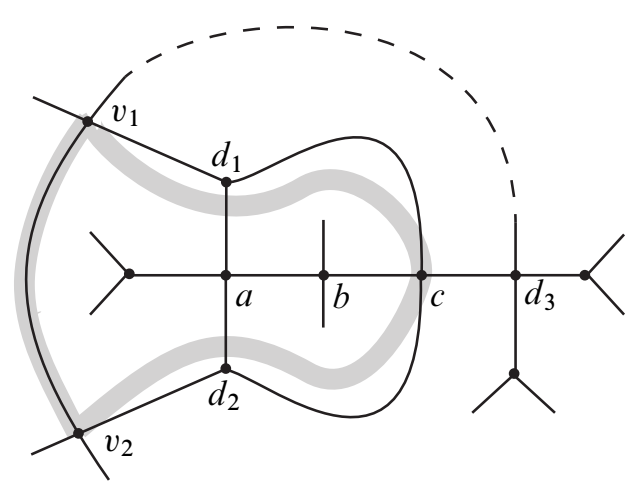

(b)

Figure 6: Some nonintrinsically knotted cases

Last, assume that $\left|V_{3}(c)\right| \geq 2$ and let $d_{1}$ and $d_{2}$ be two such vertices. As in the previous case, we may say that $d_{1}$ and $d_{2}$ are in $V(a)$, and $V\left(d_{i}\right)=\left\{a, c, v_{i}\right\}$ for $i=1,2$ where $v_{i}$ has degree 4 . When $v_{1}=v_{2},\left|V_{3}(a)\right|=3,\left|V_{4}(a, c)\right|=1$, and $v_{1}$ has degree 2 when we construct $\widehat{G}_{a, c}$, implying $\left|\widehat{E}_{a, c}\right| \leq 8$. When $\operatorname{dist}\left(v_{1}, v_{2}\right) \geq 2$, three cases occur as follows: $\left|V_{3}\left(v_{1}\right)\right| \geq 3,\left|V_{3}\left(v_{2}\right)\right| \geq 3$, or for both $i=1,2\left|V_{3}\left(v_{i}\right)\right|=2$ and $V_{4}\left(v_{i}\right)=V_{4} \backslash\left\{a, c, v_{1}, v_{2}\right\}$. All three cases satisfy that $N V_{3}\left(v_{1}, v_{2}\right)+\left|V_{4}\left(v_{1}, v_{2}\right)\right| \geq 4$, implying $\left|\widehat{E}_{v_{1}, v_{2}}\right| \leq 9$. But $\widehat{G}_{v_{1}, v_{2}}$ has a bigon containing vertices $a$ and $c$. Finally, when $\operatorname{dist}\left(v_{1}, v_{2}\right)=1$, two cases occur as follows. If $d_{3}$ has degree 3 , then by the same reason as before we may say that $d_{3}$ is also in $V(a)$, and $V\left(d_{3}\right)=\left\{a, c, v_{3}\right\}$ where $v_{3}$ has degree 4 . By the previous argument any pair of $v_{1}, v_{2}$ and $v_{3}$ has distance 1 . This 
implies that $G$ contains a triangle. If $d_{3}$ has degree 4 , then $\left|V_{3}\left(d_{3}\right)\right| \geq 2$, because at most one vertex of $V\left(d_{3}\right)$ can be $v_{1}$ or $v_{2}$. Thus, $N V_{3}\left(a, d_{3}\right) \geq 4$, implying $\left|\widehat{E}_{a, d_{3}}\right| \leq 9$. But $\widehat{G}_{a, d_{3}}$ has a triangle containing vertices $c, v_{1}$ and $v_{2}$. See Figure 6(b).

Claim 2 If $H$ is not a cycle with 6 edges, then $G$ is not intrinsically knotted.

Proof By Claim 1, if $H$ is not a cycle with 6 edges, then $H$ contains a cycle with 4 or 5 edges. First assume that $H$ contains a cycle with 5 edges. Let $\left\{a_{1}, \ldots, a_{5}\right\}$ be the set of five vertices of the cycle appearing in clockwise order. If the remaining vertex $b$ of $V_{4}$ is contained in some $V\left(a_{i}\right)$, say $i=1$, then $b$ must have distance 1 from one of $a_{3}$ and $a_{4}$, say $a_{3}$, by Claim 1. See Figure 7. If $V_{3}\left(a_{2}\right) \neq V_{3}(b)$, $N V_{3}\left(a_{2}, b\right)+\left|V_{4}\left(a_{2}, b\right)\right| \geq 5$, implying $\left|\widehat{E}_{a_{2}, b}\right| \leq 8$. Otherwise, $V_{3}\left(a_{2}\right)=V_{3}(b)$. Let $c_{1}$ and $c_{3}$ be the vertices of $V_{3}\left(a_{1}\right)$ and $V_{3}\left(a_{3}\right)$, respectively. If $c_{1}=c_{3}$, we still have $\left|\widehat{E}_{a_{2}, b}\right| \leq 9$ and $\widehat{G}_{a_{2}, b}$ has a triangle containing vertices $a_{5}, a_{4}$ and $c_{1}=c_{3}$. If $c_{1} \neq c_{3}$, then $\left|\widehat{E}_{a_{1}, a_{3}}\right| \leq 9$ and $\widehat{G}_{a_{1}, a_{3}}$ has a bigon as in the figure.

If $b$ is not contained in $V\left(a_{i}\right)$ for any $i=1, \ldots, 5$, then $\left|V_{3}\left(a_{i}\right)\right|=2$. If there is a pair of vertices $a_{i}$ and $a_{i+2}$ (or $a_{i-3}$ if $\left.i=4,5\right)$ such that $V_{3}\left(a_{i}\right)$ and $V_{3}\left(a_{i+2}\right)$ are disjoint, then $N V_{3}\left(a_{i}, a_{i+2}\right)+\left|V_{4}\left(a_{i}, a_{i+2}\right)\right|=5$, implying $\left|\widehat{E}_{a_{i}, a_{i+2}}\right| \leq 8$. Otherwise, for any pair of vertices $a_{i}$ and $a_{i+2}$ (or $a_{i-3}$ if $i=4,5$ ), $V_{3}\left(a_{i}\right)$ and $V_{3}\left(a_{i+2}\right)$ share vertices. Then they must share only one vertex as in Figure 7(b). Since there is only one extra vertex $b$ of degree 4 , for some pair of vertices $a_{i}$ and $a_{i+2}, N V_{3}\left(a_{i}, a_{i+2}\right)+$ $\left|V_{4}\left(a_{i}, a_{i+2}\right)\right|=4$ and $V_{Y}\left(a_{i}, a_{i+2}\right) \geq 1$, implying $\left|\widehat{E}_{a_{i}, a_{i+2}}\right| \leq 8$.

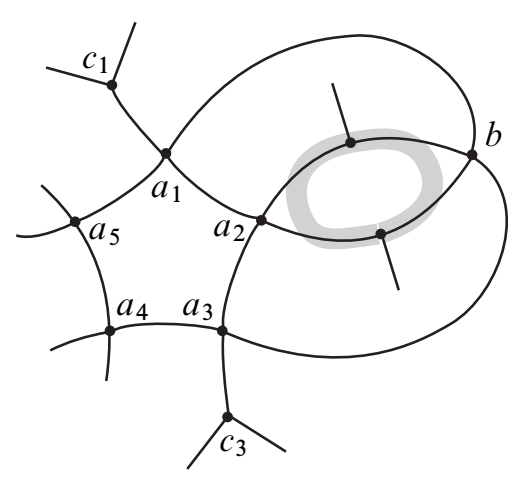

(a)

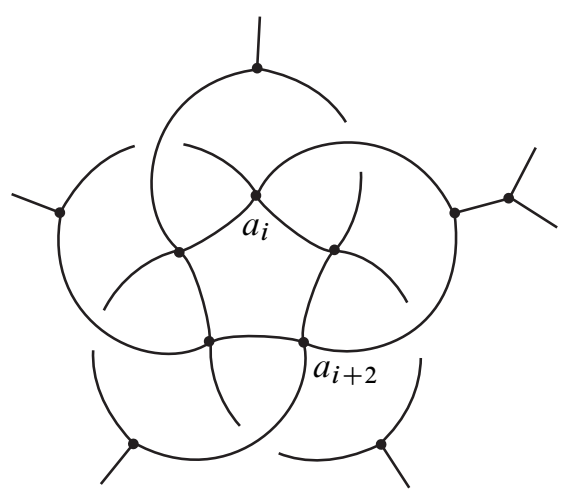

(b)

Figure 7: Cycle with 5 edges

Now, assume that $H$ contains a cycle with 4 edges. Let $\left\{a_{1}, \ldots, a_{4}\right\}$ be the set of four vertices of the cycle appearing in clockwise order. If $V\left(a_{1}\right)$ and $V\left(a_{3}\right)$ (or similarly for $V\left(a_{2}\right)$ and $\left.V\left(a_{4}\right)\right)$ share only two vertices, $a_{2}$ and $a_{4}$, then the remaining two 
vertices of $V_{4}$ must be contained in $V\left(a_{1}\right) \cup V\left(a_{3}\right)$. Otherwise, since $V\left(a_{1}\right) \cup V\left(a_{3}\right)$ has four more vertices other than $a_{2}$ and $a_{4}, N V_{3}\left(a_{1}, a_{3}\right) \geq 3$ and $\left|V_{4}\left(a_{1}, a_{3}\right)\right|=2$, implying $\left|\widehat{E}_{a_{1}, a_{3}}\right| \leq 8$. By Claim 1 , the two vertices have distance 1 , so $H$ contains a cycle with 5 edges which was dealt in the previous case. If $V\left(a_{1}\right)$ and $V\left(a_{3}\right)$ (or similarly for $V\left(a_{2}\right)$ and $\left.V\left(a_{4}\right)\right)$ share exactly three vertices, $a_{2}, a_{4}$ and $b$, then let $c_{1}$ and $c_{3}$ be the remaining vertices of $V\left(a_{1}\right)$ and $V\left(a_{3}\right)$, respectively. If both $c_{1}$ and $c_{3}$ have degree 3 , then $N V_{3}\left(a_{1}, a_{3}\right)+\left|V_{4}\left(a_{1}, a_{3}\right)\right| \geq 5$. If both have degree 4, then $H$ contains a cycle with 5 edges as in the previous case. Finally, if only $c_{1}$ (or similarly $c_{3}$ ) has degree 4 , then, by Claim $1, V\left(c_{1}\right)$ contains another vertex, say $d$, of $V_{4}$, and also $d$ must have distance 1 from one of $a_{2}$ and $a_{4}$, say $a_{4}$, as in Figure 8(a). So $N V_{3}\left(a_{4}, c_{1}\right)+\left|V_{4}\left(a_{4}, c_{1}\right)\right| \geq 4$, implying $\left|\widehat{E}_{a_{4}, c_{1}}\right| \leq 9$, and $\widehat{G}_{a_{4}, c_{1}}$ has a triangle containing vertices $a_{2}, a_{3}$, and $b$. Now we may assume that $V\left(a_{1}\right)=V\left(a_{3}\right)$ and $V\left(a_{2}\right)=V\left(a_{4}\right)$. Then $N V_{3}\left(a_{1}, a_{3}\right)+\left|V_{4}\left(a_{1}, a_{3}\right)\right|=4$, implying $\left|\widehat{E}_{a_{1}, a_{3}}\right| \leq 9$, and so $\widehat{G}_{a_{1}, a_{3}}$ has a bigon as in Figure $8(\mathrm{~b})$.

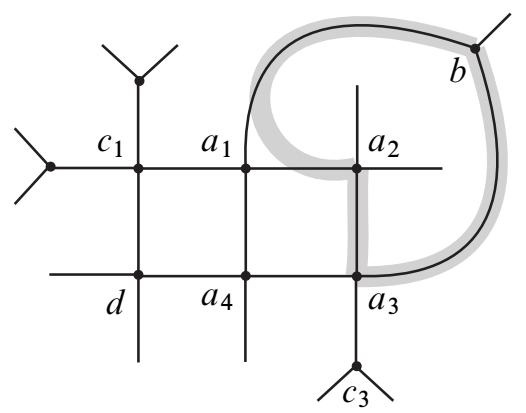

(a)

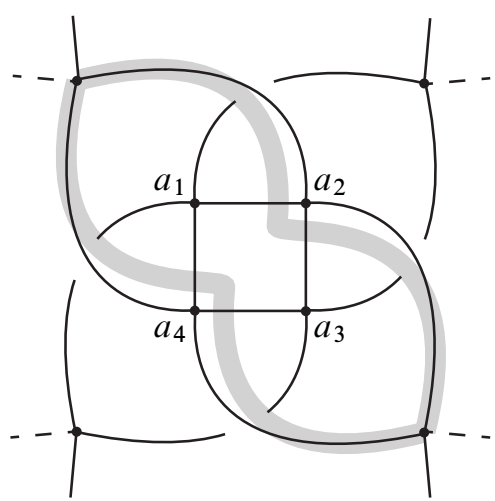

(b)

Figure 8: Cycle with 4 edges

By Claim 2, $H$ is exactly a cycle with 6 edges. Let $\left\{a_{1}, \ldots, a_{6}\right\}$ be the set of six vertices of the cycle with $a_{i}$ adjacent to $a_{i+1}$ for $i=1, \ldots, 5$, and $a_{6}$ adjacent to $a_{1}$. First, suppose that there is not a vertex $b$ in $V_{3}$ such that $V(b)=\left\{a_{1}, a_{3}, a_{5}\right\}$. If $V_{3}\left(a_{1}\right)$ and $V_{3}\left(a_{3}\right)$ are disjoint, then $N V_{3}\left(a_{1}, a_{3}\right)+\left|V_{4}\left(a_{1}, a_{3}\right)\right|=5$. If $V_{3}\left(a_{1}\right)$ and $V_{3}\left(a_{3}\right)$ share exactly one vertex $c$, then the vertex of $V(c) \backslash\left\{a_{1}, a_{3}\right\}$ is not $a_{5}$, so it should be one of $V_{Y}\left(a_{1}, a_{3}\right)$. Thus $N V_{3}\left(a_{1}, a_{3}\right)+\left|V_{4}\left(a_{1}, a_{3}\right)\right|+\left|V_{Y}\left(a_{1}, a_{3}\right)\right|=5$. If $V_{3}\left(a_{1}\right)$ and $V_{3}\left(a_{3}\right)$ are same, then $N V_{3}\left(a_{1}, a_{5}\right)+\left|V_{4}\left(a_{1}, a_{5}\right)\right|=5$, because $V_{3}\left(a_{1}\right)$ and $V_{3}\left(a_{5}\right)$ are disjoint. All three cases guarantee that $G$ is not intrinsically knotted. Therefore we may assume that there are two vertices $b_{1}$ and $b_{2}$ so that $V\left(b_{1}\right)=\left\{a_{1}, a_{3}, a_{5}\right\}$ and $V\left(b_{2}\right)=\left\{a_{2}, a_{4}, a_{6}\right\}$. See Figure 9(a). 
Suppose that there is a vertex $c$, with $c \neq b_{1}$, so that $V(c)$ contains $a_{1}$ and $a_{3}$. Let $d_{2}$ and $d_{5}$ be the vertices of $V_{3}\left(a_{2}\right)$ and $V_{3}\left(a_{5}\right)$, other than $b_{1}$ and $b_{2}$, respectively. If $d_{2} \neq d_{5}$, then $N V_{3}\left(a_{2}, a_{5}\right)=4$. If $d_{2}=d_{5}$, then $N V_{3}\left(a_{2}, a_{5}\right)=3$ and $V_{Y}\left(a_{2}, a_{5}\right)$ is not empty. Both cases provide $\left|\widehat{E}_{a_{2}, a_{5}}\right| \leq 9$, and $\widehat{G}_{a_{2}, a_{5}}$ has a triangle containing vertices $a_{1}, a_{3}$, and $c$. Therefore we may assume in general that for any vertex $c$, except $b_{1}$ and $b_{2}, V(c)$ does not contain both $a_{i}$ and $a_{i+2}$ for any $i=1,2,3,4$, and both $a_{i}$ and $a_{i-4}$ for any $i=5,6$.

Now we conclude $E \backslash\left\{E^{2}\left(b_{1}\right) \cup E^{2}\left(b_{2}\right)\right\}$ consists of three extra edges. Note that each vertex of these edges has degree 3 , and there are four more vertices of degree 3 besides $b_{1}$ and $b_{2}$. These two facts guarantee that these extra edges must be connected as a tree. This tree can be of two types; either all three edges are incident to one vertex $d$, or two edges are incident to different endpoints of the other edge $e$, respectively. In both cases, any two edges adjoined to the tree at the same vertex at the end must be also incident to $a_{i}$ and $a_{i+3}$, respectively, for some $i=1,2,3$. Therefore, $G$ is one of three graphs as in Figure 9(b)-(c), depending on the type of the tree. The graph $G$ in Figure 9(b) is $H_{12}$, which is intrinsically knotted. But the two graphs in Figure 9(c) are not intrinsically knotted because, for some $i,\left|\widehat{E}_{a_{i}, a_{i+2}}\right| \leq 9$, and $\widehat{G}_{a_{i}, a_{i+2}}$ has a triangle.

\subsection{Case $\left(\left|V_{4}\right|,\left|V_{3}\right|\right)=(9,2)$}

Let $b_{1}$ and $b_{2}$ be the vertices of $V_{3}$. Since $\left|V_{3}\right|=2$, there are at least three vertices, $a_{1}$, $a_{2}$, and $a_{3}$, in $V_{4}$ such that all vertices of each $V\left(a_{i}\right)$ have degree 4 . If $\operatorname{dist}\left(a_{1}, a_{2}\right)=1$, then $V\left(a_{1}\right) \cup V\left(a_{2}\right)$ consists of 8 vertices of $V_{4}$, and so let $c$ be the ninth vertex. Let $d$ be any vertex among $V\left(a_{1}\right) \cup V\left(a_{2}\right) \backslash\left\{a_{1}, a_{2}\right\}$ which is not contained in $V(c)$. We assume that $d$ is in $V\left(a_{1}\right)$. Then $V(d)$ should be contained in $V\left(a_{2}\right) \cup\left\{b_{1}, b_{2}\right\}$. This implies that $N E\left(a_{2}, d\right)=8$ and $\left|V_{3}(d)\right|+\left|V_{4}\left(a_{2}, d\right)\right| \geq 4$, implying $\left|\widehat{E}_{a_{2}, d}\right| \leq 9$. Since $c$ has degree 4 in $\widehat{G}_{a_{2}, d}$, it follows that $\widehat{G}_{a_{2}, d}$ is not homeomorphic to $K(3,3)$. We have the same result for any choices of pairs among $a_{1}, a_{2}$, and $a_{3}$.

Now assume that the distance between any pair among $a_{1}, a_{2}$, and $a_{3}$ is at least 2 . We separate into several cases according to the number $\left|V_{4}\left(a_{1}, a_{2}\right)\right|$. If $V_{4}\left(a_{1}, a_{2}\right)=$ $\varnothing$ (ie $\left.\operatorname{dist}\left(a_{1}, a_{2}\right)>2\right)$, then $\left|V_{4}\right| \geq 10$, a contradiction. If $V_{4}\left(a_{1}, a_{2}\right)=\{d\}$, then $V_{4}=V\left(a_{1}\right) \cup V\left(a_{2}\right) \cup\left\{a_{1}, a_{2}\right\}$. This implies that $a_{3} \in V\left(a_{1}\right) \cup V\left(a_{2}\right)$, so $\operatorname{dist}\left(a_{1}, a_{3}\right)=1$ or $\operatorname{dist}\left(a_{2}, a_{3}\right)=1$, both of which were dealt with in the previous case. If $V_{4}\left(a_{1}, a_{2}\right)=\left\{d_{1}, d_{2}\right\}$, then $V\left(d_{1}\right) \cup V\left(d_{2}\right) \backslash\left\{a_{1}, a_{2}\right\}$ is contained in $\left\{a_{3}, b_{1}, b_{2}\right\}$. This implies that each $V\left(d_{i}\right) \backslash\left\{a_{1}, a_{2}\right\}$ is a set of two vertices among $\left\{a_{3}, b_{1}, b_{2}\right\}$, so that $\left|V_{3}\left(d_{1}, d_{2}\right)\right|+\left|V_{4}\left(d_{1}, d_{2}\right)\right| \geq 4$, implying $\left|\widehat{E}_{d_{1}, d_{2}}\right| \leq 9$. Since at least two of four vertices in $V\left(a_{1}\right) \cup V\left(a_{2}\right) \backslash\left\{d_{1}, d_{2}\right\}$ still have degree 4 in $\widehat{G}_{d_{1}, d_{2}}$, it follows 


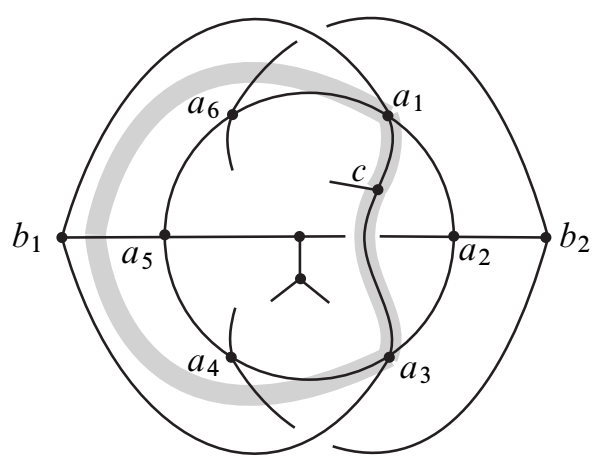

(a)

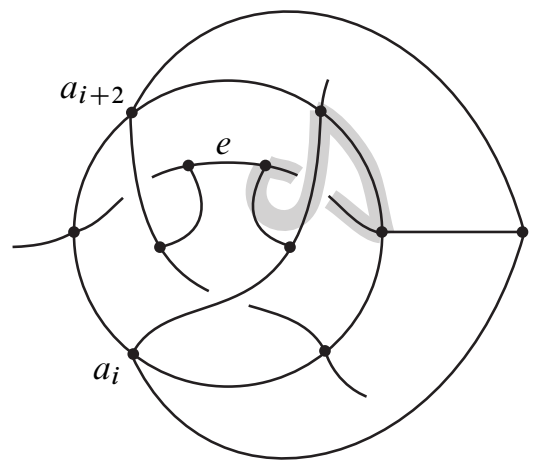

(c)

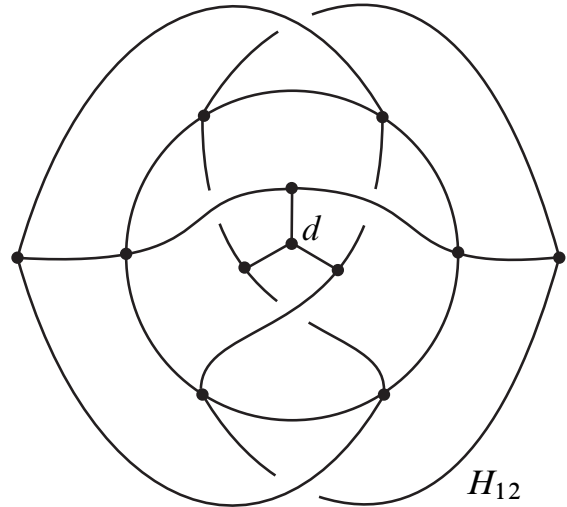

(b)

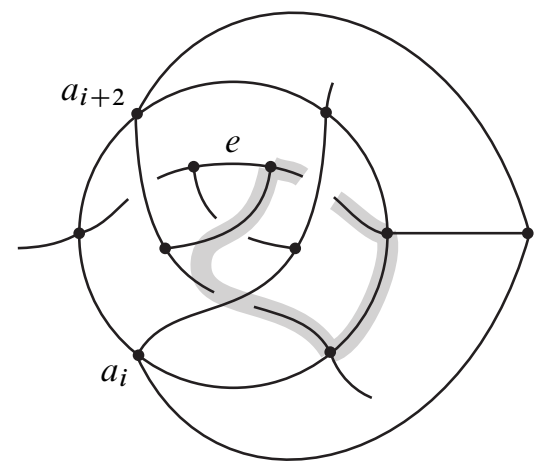

Figure 9: Constructing $H_{12}$

that $\widehat{G}_{d_{1}, d_{2}}$ is not homeomorphic to $K(3,3)$. If $V_{4}\left(a_{1}, a_{2}\right)=\left\{d_{1}, d_{2}, d_{3}\right\}$, then $V\left(d_{1}\right) \cup V\left(d_{2}\right) \cup V\left(d_{3}\right) \backslash\left\{a_{1}, a_{2}\right\}$ is contained in $\left\{a_{3}, a_{4}, b_{1}, b_{2}\right\}$, where $a_{3}$ and $a_{4}$ are the remaining two vertices of degree 4 other than $V\left(a_{1}\right) \cup V\left(a_{2}\right) \cup\left\{a_{1}, a_{2}\right\}$. Thus each $V\left(d_{i}\right) \backslash\left\{a_{1}, a_{2}\right\}$ is the set of two vertices among $\left\{a_{3}, a_{4}, b_{1}, b_{2}\right\}$. This implies that $\left|V_{3}\left(d_{i}, d_{j}\right)\right|+\left|V_{4}\left(d_{i}, d_{j}\right)\right| \geq 4$ for some $i, j=1,2,3$, implying $\left|\widehat{E}_{d_{i}, d_{j}}\right| \leq 9$. Since at least one of three vertices $V\left(a_{1}\right) \cup V\left(a_{2}\right) \backslash\left\{d_{i}, d_{j}\right\}$ still has degree 4 in $\widehat{G}_{d_{i}, d_{j}}$, it follows that $\widehat{G}_{d_{i}, d_{j}}$ is not homeomorphic to $K(3,3)$. Finally, if $\left|V_{4}\left(a_{1}, a_{2}\right)\right|=4$, then $\left|\widehat{E}_{a_{1}, a_{2}}\right| \leq 9$. Since $\widehat{G}_{a_{1}, a_{2}}$ still has the remaining three vertices of degree 4 , it follows that $\widehat{G}_{a_{1}, a_{2}}$ is not homeomorphic to $K(3,3)$.

\section{$5 \operatorname{deg}(a)=3$}

Since we are working on the graph with 21 edges and every vertex has degree 3 , there are exactly 14 vertices. First, suppose that there exists a pair of vertices $a$ and $b$ with 
$\operatorname{dist}(a, b) \geq 4$. Then $E^{2}(a)$ and $E^{2}(b)$ can share vertices, but they do not share edges in common. Since $\left|E^{2}(a) \cup E^{2}(b)\right|=18$ and $|V(a) \cup V(b) \cup\{a, b\}|=8$, the 18 endpoints of $E^{2}(a), E^{2}(b)$, and three extra edges which are $E \backslash\left\{E^{2}(a) \cup E^{2}(b)\right\}$, meet at six vertices. If any two edges of $E^{2}(a) \backslash E(a)$ (and similarly for $b$ ) are incident to one vertex $c$ of these six vertices, take the unique vertex $d$ of $V(a)$ which is not an endpoint of these two edges. Then $N E(b, d)=6$ and $N V_{3}(b, d)=6$, implying $\left|\widehat{E}_{b, d}\right|=9$. But $\widehat{G}_{b, d}$ has a triangle containing $c$ and the two vertices of $V(a) \backslash\{d\}$, so it follows that $\widehat{G}_{b, d}$ is not homeomorphic to $K(3,3)$. If not, each of these six vertices is a common endpoint of one edge of $E^{2}(a)$, one edge of $E^{2}(b)$, and one extra edge. Now, take an extra edge $e$ and let $b_{1}$ and $b_{2}$ be the two vertices of $V(b)$ which have distance 1 from the endpoints of $e$. Let $b_{3}$ be the remaining vertex of $V(b)$. Then $N E\left(b_{1}, b_{2}\right)=6, N V_{3}\left(b_{1}, b_{2}\right)=5$, and $V_{Y}\left(b_{1}, b_{2}\right)=\left\{b_{3}\right\}$, implying $\left|\widehat{E}_{b_{1}, b_{2}}\right|=9$. But $\widehat{G}_{b_{1}, b_{2}}$ has a triangle containing $a$ and two vertices of $V(a)$, so it follows that $\widehat{G}_{b_{1}, b_{2}}$ is not homeomorphic to $K(3,3)$. See Figure $10(\mathrm{a})$.

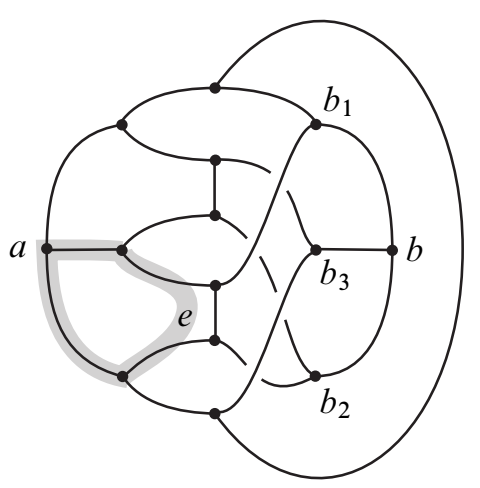

(a)

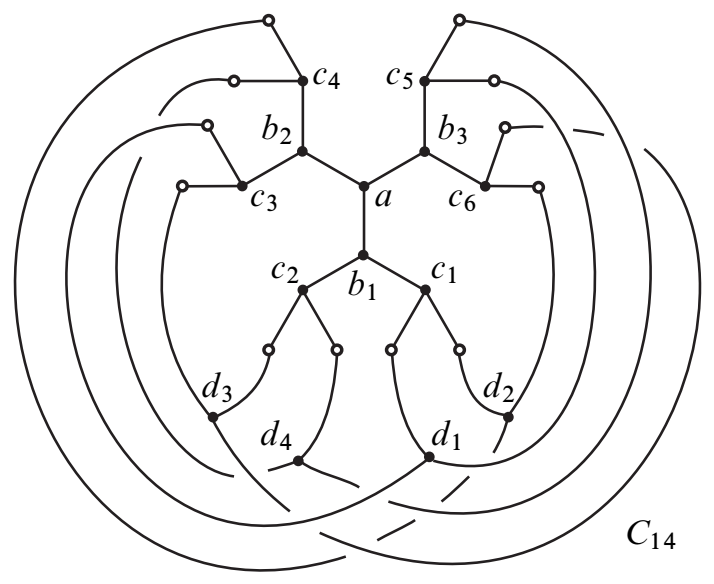

(b)

Figure 10: Constructing $C_{14}$

Therefore, we assume that the distance between any pair of vertices cannot exceed 3 . Now we construct the intrinsically knotted graph $G$ satisfying these conditions. Take a vertex $a$ and let $V(a)=\left\{b_{1}, b_{2}, b_{3}\right\}$ and $V\left(b_{i}\right)=\left\{a, c_{2 i-1}, c_{2 i}\right\}$ for $i=1,2,3$. As in Figure 10(b), the graph $E(a) \cup E\left(c_{1}\right) \cup \cdots \cup E\left(c_{6}\right)$ consists of 21 edges and 22 vertices. We show this is the only way to draw the graph with 21 edges such that all vertices have distance at most 3 from $a$ and 10 vertices $a, b_{1}, b_{2}, b_{3}, c_{1}, \ldots, c_{5}$, and $c_{6}$ have degree 3. Now we join 12 white dots in Figure 10(b) into 4 groups indicating the remaining 4 vertices by $d_{1}, d_{2}, d_{3}$ and $d_{4}$. Thus each $V\left(d_{j}\right), j=1,2,3,4$, has three vertices among $c_{1}, \ldots, c_{6}$. Since the distance between any $c_{i}$ and $c_{i^{\prime}}$ cannot 
exceed 3, the following two properties must be satisfied. The first property is that $V\left(d_{j}\right)$ contains exactly one vertex from each group $\left\{c_{2 i-1}, c_{2 i}\right\}$ for $i=1,2,3$. For example, if $V\left(d_{1}\right)=\left\{c_{1}, c_{2}, c_{3}\right\}$ (ie two vertices from the group $\left\{c_{1}, c_{2}\right\}$ ), then we can connect $c_{1}$ to at most two vertices among $\left\{c_{4}, c_{5}, c_{6}\right\}$ through some $E\left(d_{j}\right)$. This means that the distance between $c_{1}$ and one among $\left\{c_{4}, c_{5}, c_{6}\right\}$ exceeds 3 . The second property is that different $V\left(d_{j}\right)$ and $V\left(d_{j^{\prime}}\right)$ share at most one vertex. For example, if they share two vertices $c_{1}$ and $c_{3}$, then $\operatorname{dist}\left(c_{1}, c_{4}\right)=4$. From these two properties, without loss of generality, we may say that

$$
\begin{array}{ll}
V\left(d_{1}\right)=\left\{c_{1}, c_{3}, c_{5}\right\}, & V\left(d_{2}\right)=\left\{c_{1}, c_{4}, c_{6}\right\}, \\
V\left(d_{3}\right)=\left\{c_{2}, c_{3}, c_{6}\right\}, & V\left(d_{4}\right)=\left\{c_{2}, c_{4}, c_{5}\right\}
\end{array}
$$

as drawn in Figure 10(b). This graph is exactly $C_{14}$.

\section{References}

[1] P Blain, G Bowlin, T Fleming, J Foisy, J Hendricks, J Lacombe, Some results on intrinsically knotted graphs, J. Knot Theory Ramifications 16 (2007) 749-760 MR2341314

[2] J H Conway, C M Gordon, Knots and links in spatial graphs, J. Graph Theory 7 (1983) 445-453 MR722061

[3] N Goldberg, T W Mattman, R Naimi, Many, many more intrinsically knotted graphs, Algebr. Geom. Topol. 14 (2014) 1801-1823 MR3212585

[4] R Hanaki, R Nikkuni, K Taniyama, A Yamazaki, On intrinsically knotted or completely 3-linked graphs, Pacific J. Math. 252 (2011) 407-425 MR2860431

[5] B Johnson, M E Kidwell, T S Michael, Intrinsically knotted graphs have at least 21 edges, J. Knot Theory Ramifications 19 (2010) 1423-1429 MR2746195

[6] T Kohara, S Suzuki, Some remarks on knots and links in spatial graphs, from: "Knots 90", (A Kawauchi, editor), de Gruyter, Berlin (1992) 435-445 MR1177440

[7] R Motwani, A Raghunathan, H Saran, Constructive results from graph minors: Linkless embeddings, from: "Proc. $29^{\text {th }}$ annual symposium on foundations of computer science”, IEEE, Piscataway, NJ (1988) 398-409

[8] M Ozawa, Y Tsutsumi, Primitive spatial graphs and graph minors, Rev. Mat. Complut. 20 (2007) 391-406 MR2351115

[9] N Robertson, PD Seymour, Graph minors, XX: Wagner's conjecture, J. Combin. Theory Ser. B 92 (2004) 325-357 MR2099147 
ML,HK,SO: Department of Mathematics, Korea University Anam-dong, Sungbuk-ku, Seoul 136-701, South Korea

HJL: Department of Mathematical Sciences, KAIST 291 Daehak-ro, Yuseong-gu, Daejeon 305-701, South Korea

mjmlj@korea.ac.kr, kimhjun@korea.ac.kr, hjwith@kaist.ac.kr, seungsang@korea.ac.kr

Received: 9 June 2014 Revised: 13 March 2015 